\title{
Preditores de abandono de tratamento na psicoterapia psicanalítica de crianças
}

\author{
Predictors of treatment dropout in child psychoanalytical psychotherapy
}

\author{
Marina Bento Gastaud ${ }^{1}$, Maria Lúcia Tiellet Nunes² \\ ${ }^{1}$ Especialista em Teoria Psicanalítica e Psicoterapias da Infância e Adolescência, Contemporâneo Instituto de Psicanálise e Transdisciplinaridade, Porto Alegre, RS. \\ Mestre em Psicologia Clínica, Pontifícia Universidade Católica do Rio Grande do Sul (PUCRS), Porto Alegre, RS. Doutoranda em Ciências Médicas: Psiquiatria, \\ Universidade Federal do Rio Grande do Sul (UFRGS), Porto Alegre, RS. ${ }^{2}$ Doutora em Psicologia: Tratamento e Prevenção, Freie Universität Berlin, Berlim, Alemanha. \\ Professora titular, PUCRS. \\ Coordenação de Aperfeiçoamento de Pessoal de Nível Superior (CAPES).
}

\section{Resumo}

Introdução: As altas taxas de abandono em psicoterapia e a lacuna na literatura sobre abandono de tratamento na psicoterapia de crianças justificam a realização de pesquisas com esse foco. A literatura aponta que algumas variáveis sociodemográficas e clínicas poderiam predizer o desfecho da psicoterapia. O presente estudo objetivou verificar preditores de abandono de tratamento na psicoterapia psicanalítica de crianças na amostra pesquisada.

Método: Trata-se de um estudo documental, retrospectivo, com os prontuários de duas instituições de atendimento psicológico a crianças em Porto Alegre. Resultados: Foram pesquisados prontuários de 2.106 crianças. Dessas, 200 tiveram alta e 793 abandonaram seus atendimentos. Os grupos foram comparados, e os resultados indicam que meninos apresentam mais risco de abandonar o tratamento; já crianças encaminhadas por neurologistas ou por psicólogos apresentam menos risco de abandono. Após o sexto mês de atendimento, o risco de abandono decai consideravelmente.

Discussão: Os resultados são discutidos à luz da literatura sobre gênero, abandono de tratamento e crianças em psicoterapia. Algumas hipóteses são levantadas para as associações e não-associações encontradas neste estudo.

Conclusões: Conhecer preditores de abandono em psicoterapia possibilita aos terapeutas identificar precocemente pacientes pertencentes ao grupo de risco para abandono, oportunizando-lhes trabalhar preventivamente e mais diretamente aspectos de resistência e transferência negativa desses pacientes e seus familiares, principalmente nos primeiros 6 meses de tratamento. Criar técnicas de intervenção precoce com os pais de tais crianças e realizar tratamentos transdisciplinares também são saídas possíveis para evitar o abandono.

Descritores: Abandono, psicoterapia psicanalítica, crianças.

\begin{abstract}
Introduction: The high rates of dropout in psychotherapy and the shortage of studies on treatment dropout in child psychotherapy are strong reasons for further research on this topic. The literature suggests that some sociodemographic and clinical variables could predict psychotherapy outcome. The objective of the present study was to identify predictors of treatment dropout in child psychotherapy.

Methods: This is a retrospective study involving the analysis of medical records of two institutions that provide psychological care to children in Porto Alegre, RS, Brazil.

Results: We analyzed the medical records of 2,106 children. Of these, 200 children were discharged from treatment and 793 dropped out. The two groups were compared, and the results suggest that boys have higher risk of dropping out, while children referred by neurologists or psychologists are at a lower risk of dropping out. After six months of treatment, the dropout risk is considerably reduced.

Discussion: The results are discussed based on the literature on gender, treatment dropout and child psychotherapy. Some hypotheses were proposed to explain the associations and lack of associations found in the present study.

Conclusions: Knowing predictors of dropout from psychotherapy makes it possible for therapists to identify patients belonging to the risk group in the beginning of treatment. Therefore, they can try to directly prevent aspects related to resistance and negative transference of these patients and their relatives, mainly during the first 6 months of treatment. Implementing early intervention techniques with the parents and delivering transdisciplinary therapies are possible solutions to avoid dropout.
\end{abstract}

Keywords: Dropout, analytical psychotherapy, children.

\section{Correspondência:}

Marina Bento Gastaud, PUCRS, Avenida Ipiranga, 6681, prédio 11, $9^{\circ}$ andar, sala 928, CEP 90619-900, Porto Alegre, RS. E-mail: marina.gastaud@yahoo.com

Não há conflitos de interesse associados à publicação deste artigo.

Copyright (C) Revista de Psiquiatria do Rio Grande do Sul - APRS

Recebido 19/09/2008. Aceito em 28/11/2008. 


\section{Introdução}

O abandono em psicoterapia - seja de crianças, adolescentes ou adultos - tem amplo espaço de pesquisa e discussão no meio clínico e científico, visto apontarem vários estudos para uma taxa entre 25 e $60 \%$ dos casos pesquisados ${ }^{1-4}$, dependendo do estudo e das características do serviço.

Dentre as pesquisas sobre abandono de tratamento, a maior parte se refere a pacientes adultos, existindo lacunas na literatura nacional e internacional no que diz respeito a abandono na psicoterapia de crianças. No que se refere a estudos sobre abandono em psicoterapia, apenas 1 a $2 \%$ investigam crianças ou adolescentes ${ }^{5}$. $\mathrm{O}$ fato de as pesquisas contarem principalmente com amostras de adultos aponta para a necessidade de se investigarem crianças, pois os fatores relacionados ao abandono de terapia por parte de adultos podem não ser aplicáveis a crianças. Por exemplo, alguns tipos de diferença entre paciente e terapeuta envolvendo características sociodemográficas (tais como paciente do sexo masculino sendo atendido por terapeuta do sexo feminino ou paciente mais velho sendo atendido por terapeuta muito jovem) constituem-se em preditores importantes para o abandono no caso da psicoterapia de adultos, mas o mesmo não se mostra verdadeiro no caso de crianças, conforme Midgley \& Navridi ${ }^{4}$. Faltam, portanto, dados que especifiquem os fatores que podem interferir no fenômeno do abandono na psicoterapia de crianças. Entender os fatores que contribuem para o abandono de tratamento parece importante, tanto para formar uma base para identificação de casos em risco quanto para intervir no sentido de melhor engajar a família no tratamento ${ }^{5}$.

Há, dessa forma, necessidade de seguir investigando as características do paciente que o influenciam na decisão de interromper o tratamento, pois é frequente que nem mesmo ele próprio saiba manifestamente os fatores envolvidos em sua decisão. Na maior parte das vezes, os pacientes não verbalizam nenhuma razão para o abandono do tratamento ou, quando a referem, informam dificuldades financeiras ${ }^{6}$.

Em pacientes adultos, muitos estudos encontraram características clínicas associadas ao abandono, tais como: aliança terapêutica; troca de psicoterapeutas; número de faltas durante o tratamento ${ }^{7}$; características psicopatológicas, especificamente "psicoticismo" e "sentimentalismo"; motivação e sensibilidade interpessoal"; sintomas psicossomáticos; sexualidade insatisfatória; abuso de álcool e substâncias farmacêuticas; e exposição na infância à violência e ao abuso sexual ${ }^{10}$. Ainda em estudos concentrados em adultos, dados sociodemográficos também mostraram associações com abandono, dentre eles: local de residência do paciente (fora da área do serviço), falta de vale transporte, o fato de o paciente ter procurado espontaneamente o serviço, o paciente ser do sexo masculino ${ }^{11}$, a idade do paciente e o fato de este ter filhos?

Em adolescentes, Pelkonen et al. ${ }^{12}$ investigaram pacientes entre 12 e 18 anos que abandonaram o tratamento, tanto no que diz respeito a dados clínicos como a dados sociodemográficos.
Os autores observaram associação entre abandono precoce e baixo status socioeconômico dos pais, o paciente não apresentar transtornos de humor, não usar medicação psiquiátrica e fazer uso abusivo de substâncias.

Ao contrário do que ocorre com as pesquisas em psicoterapia realizadas com adultos, os estudos de saúde mental realizados com crianças são escassos, sendo que a maior parte dos dados colhidos nessa área refere-se à população escolar. É, no entanto, preocupante a magnitude dos problemas de saúde mental em crianças. A partir de recente estudo ${ }^{13}$, Nunes et al. ${ }^{14}$ estimaram que $10 \%$ da população de Porto Alegre entre 0 e 9 anos de idade precisariam de atendimento especializado em saúde mental.

Embora sejam poucos os estudos, a literatura mostra a existência de preditores sociodemográficos e clínicos de abandono na psicoterapia de crianças. Os fatores que mais atraem a atenção dos pesquisadores, nos casos de psicoterapia de crianças, incluem: status socioeconômico, fonte de encaminhamento, atraso na espera para atendimento, distância geográfica dos serviços de atendimento, história prévia de tratamento, estresse parental, expectativas dos pais, natureza da psicopatologia da criança, sexo da criança, idade da criança e dos pais, origens étnicas, idioma falado em casa, desemprego, educação da mãe, configuração parental e familiar, renda, tamanho da família e duração da queixa da criança ${ }^{3-5}$. Os achados quanto ao impacto desses fatores são contraditórios, e as evidências sugerem que nenhum fator isolado pode ser necessário ou suficiente para predizer o abandono de tratamento com crianças. Mesmo assim, Luk et al. ${ }^{3}$ referem que o abandono parece estar associado à configuração familiar (mães jovens e famílias monoparentais), situação socioeconômica (famílias socioeconomicamente prejudicadas) e motivo de consulta (crianças com grave comportamento antissocial). Kazdin ${ }^{5}$, por sua vez, sugere associação positiva e direta entre abandono e desvantagem socioeconômica da família (menor educação, menor renda, receber ajuda financeira do governo e/ou moradia em locais muito pequenos ou perigosos), constelação familiar (mães mais jovens, famílias compostas por apenas um dos pais, famílias formadas por grupos minoritários), psicopatologia dos pais (mãe relata história de comportamento antissocial quando era criança), sintomas das crianças (alto número de sintomas agressivos, antissociais e de conduta na criança, independente do diagnóstico) e funcionamento acadêmico/educacional da criança (abaixo da inteligência normal, história de repetências escolares). Midgley \& $\mathrm{Navridi}^{4} \mathrm{de}^{-}$ monstraram que o tempo de atendimento está associado ao abandono: crianças que permaneceram em atendimento por mais de 2 anos tendem a não abandonar seus tratamentos. Tais autores ainda encontraram associação entre abandono e sexo, sendo crianças do sexo feminino mais prováveis de concluir seus atendimentos e de não abandoná-los.

Percebe-se, nesses estudos, que condições econômicas desfavoráveis do paciente ou de sua família mostram-se como um fator associado ao abandono de psicoterapia. Garcia \& 
Weisz ${ }^{15}$ pesquisaram razões para abandono de tratamento na psicoterapia de crianças e adolescentes e demonstraram que problemas no relacionamento terapêutico e questões financeiras foram os únicos fatores que distinguiram os pacientes que abandonaram dos que concluíram seus tratamentos. Em decorrência do alto custo financeiro dos tratamentos psicoterapêuticos, o atendimento em instituição aparece como uma saída para o impasse criado quando o paciente precisa de um atendimento de baixo custo. Há, entretanto, disparidade entre os tipos de serviço prestados pelas instituições e a clientela que as procuram, tendo em vista que as clínicas-escola (principais instituições prestadoras de serviços de psicoterapia à população em geral) foram organizadas de acordo com as necessidades e possibilidades dos profissionais e não necessariamente segundo demandas da população a ser atendida ${ }^{16,17}$. Tal defasagem é, possivelmente, mais uma variável associada aos altos índices de interrupção do tratamento, já que estudos comprovam que abandono está relacionado à falta de conexão entre terapeuta e paciente ${ }^{18}$. Torna-se, pois, necessária a realização de pesquisas que mapeiem o perfil dos pacientes atendidos em clínicas-escola mais associado ao abandono do tratamento, a fim de capacitar os profissionais que atendem em tais instituições a lidar com essas possíveis características.

As terapias de crianças sofrem grande influência dos pais ou responsáveis: eles decidem o momento da consulta, levam o paciente a consultar e arcam financeiramente com o tratamento. Garcia \& Weisz ${ }^{15}$, por exemplo, pesquisaram com pais de crianças e adolescentes as razões para terem interrompido o tratamento de seus filhos. Eles concluíram que problemas no relacionamento terapêutico foi o maior motivo alegado, seguido de problemas financeiros. Luk et al. ${ }^{3}$ pesquisaram os pais de crianças com problemas de conduta que abandonaram os tratamentos realizados em um serviço de saúde mental. O abandono mostrou associação com pais que apresentaram insatisfação com o serviço em que eram realizados os atendimentos bem como com pais que tinham a percepção de o tratamento ser pouco organizado. Os mesmos autores também associaram abandono com mães muito jovens e com baixa escolaridade.

Parece, entretanto, também haver características da própria criança envolvidas na questão do abandono da psicoterapia, como revelam Di Gallo et al. ${ }^{19}$. Os autores pesquisaram crianças e adolescentes atendidos em um ambulatório psiquiátrico em Basel, Suíça. Os abandonos de tratamento apresentaram maior incidência nos casos em que as crianças e os adolescentes haviam sido encaminhados por serviços de emergência e hospitais gerais e foram em menor número nos casos em que os próprios pacientes solicitaram atendimento.

Ademais, um estudo ${ }^{20}$ realizado com pais de crianças atendidas em psicoterapia se propôs a investigar se a entrega de materiais informativos aos pais aumentava as taxas de adesão ao tratamento. Foram mostrados aos pais vídeos e folhetos sobre a importância do envolvimento deles no processo, sobre como o brinquedo é usado em terapia, sobre confidencialidade e sobre a importância de persistir no tratamento até que os objetivos sejam atingidos. Os autores verificaram que os procedimentos preparatórios não aumentaram os índices de adesão. Tal achado fornece indícios, dentre outras possibilidades, de que fatores relacionados à criança também precisam ser trabalhados para que ela permaneça em psicoterapia.

O presente estudo tem, portanto, o objetivo de investigar relações entre características sociodemográficas e clínicas de crianças atendidas em instituições de psicoterapia psicanalítica e o abandono da psicoterapia. Através de resultados que contribuam para elucidar o fenômeno, medidas preventivas estratégicas poderão ser estabelecidas para diminuir as altas taxas de abandono nas psicoterapias de crianças.

\section{Objetivos}

- Examinar a relação entre variáveis sociodemográficas (idade, sexo, configuração familiar, escolaridade e cidade em que mora) e o abandono de psicoterapia psicanalítica de crianças.

- Examinar a relação entre variáveis clínicas (motivo da consulta, flonte de encaminhamento e tempo de atendimento) e o abandono de psicoterapia psicanalítica de crianças.

- Verificar quais variáveis sociodemográficas e clínicas predizem o abandono de psicoterapia psicanalítica de crianças.

\section{Método}

Trata-se de um estudo quantitativo, retrospectivo, descritivo, a partir de material documental arquivado sobre atendimento de crianças em psicoterapia psicanalítica.

A pesquisa foi realizada em duas instituições: Contemporâneo (Instituto de Psicanálise e Transdisciplinaridade) e CEAPIA (Centro de Estudos, Atendimento e Pesquisa da Infância e da Adolescência). O Contemporâneo e o CEAPIA são instituições de pós-graduação no ensino em psicanálise que formam especialistas em psicoterapia psicanalítica de crianças. As instituições contam com ambulatórios que atendem a população de baixa e média renda por um valor mais acessível do que o geralmente cobrado em consultórios particulares. Os tratamentos realizados nessas instituições não têm prazo limite para o término (término aberto) e contam com a frequência semanal estipulada pelo paciente e pelo terapeuta no início do atendimento. Os honorários dos atendimentos são determinados pelo terapeuta em conjunto com seu paciente, a partir de um valor mínimo estipulado pelas instituições. Adotou-se o princípio do Estatuto da Criança e do Adolescente (Artigo $\left.2^{\circ}, 1990\right)$ que considera criança "a pessoa com até 12 anos incompletos" ${ }^{\prime 21}$. Foram incluídas na análise todas as crianças que buscaram psicoterapia no Contemporâneo ou no CEAPIA 
entre 1979 e 2007. Foram excluídos os prontuários referentes à segunda procura por atendimento do mesmo paciente em outro momento.

O banco de dados foi composto no Statistical Package for the Social Sciences (SPSS) versão 13, com base em um formulário contendo as variáveis sociodemográficas e clínicas extraídas dos prontuários. As variáveis de interesse foram definidas conforme os critérios listados a seguir.

\section{Término do tratamento}

Definido conforme informação constante nos prontuários. Quando os terapeutas não expressavam objetivamente um desfecho claro, as informações foram interpretadas a partir dos dados presentes nos prontuários a respeito da evolução do caso de acordo com as definições listadas a seguir ${ }^{22}$.

\section{Não-aderência}

$\mathrm{O}$ atendimento é interrompido na fase de avaliação da psicoterapia, ou seja, antes que os objetivos estabelecidos para o tratamento estejam claros para ambos os participantes ou em situações em que não há indicação de tratamento. Entende-se que a avaliação tenha duração de 1 mês.

\section{Abandono}

A psicoterapia é encerrada antes que os objetivos estabelecidos no contrato tenham sido atingidos, independentemente dos motivos que levaram o paciente ou o terapeuta a interrompê-la e independentemente do fato de a decisão ter sido uni ou bilateral. $\mathrm{O}$ atendimento deve ter tido duração mínima de 1 mês para o paciente ser considerado abandonante.

\section{Alta}

A psicoterapia é encerrada quando os objetivos estabelecidos no contrato foram atingidos.

Sexo, idade, configuração familiar, escolaridade, cidade em que mora a criança e fonte de encaminhamento

Definidos conforme constante na ficha do paciente, de acordo com informações postuladas pelos pais ou responsáveis pela criança.

\section{Tempo de consulta}

Conforme informação fornecida pelo terapeuta da criança ao encerrar o atendimento, constante nos prontuários.

\section{Motivo de consulta}

Definido conforme descrição dada: a) pelos pais/responsáveis na triagem ou ao preencherem a ficha de contato inicial nas instituições; b) pelo profissional que realizou a triagem daquele paciente ao preencher a ficha de avaliação na chegada do paciente à instituição; e c) pelo terapeuta responsável pelo caso ao preencher o roteiro de avaliação psicológica do paciente. Foram coletadas e transportadas ao banco de dados as três primeiras queixas fornecidas em cada um desses momentos. As informações referentes ao motivo de consulta apresentadas no prontuário foram armazenadas de forma livre. Para classificação dessa variável foram utilizadas, via análise clínica de cada paciente, as escalas de comportamento internalizante, externalizante, neutra e social do Child Behavior Checklist (CBCL), Syndrome Scale 6-18. As escalas utilizadas compreendem:

- Ansiedade/depressão (choros, medos, não se sente amado, etc.);

- Retraimento/depressão (tímido, triste, prefere ficar sozinho, etc.);

- Queixas somáticas (tontura, cansaço, náusea, dor de cabeça, etc.);

- Problemas de relacionamento (não se dá bem com as pessoas, dependente, pessoas implicam com ele, etc.);

- Problemas do pensamento (ouve vozes, vê coisas, comportamentos estranhos, etc.);

- Problemas de atenção (não se concentra, muito agitado, devaneios, etc.);

- Comportamento desafiador/opositor (vandalismo, roubos, mentiras, etc.);

- Comportamento agressivo (brigas, gritos, discussões, etc.);

- Problemas de aprendizagem (repetência, dificuldade em alguma disciplina, etc.).

Tal análise foi realizada por um grupo de juízes que, através de um entendimento clínico das queixas constantes no banco de dados, categorizou cada paciente dentro das possibilidades propostas pelo CBCL.

Desde o início de seus tratamentos, tanto no Contemporâneo quanto no CEAPIA, os responsáveis pelas crianças assinam um termo de autorização, permitindo que os dados do seu atendimento fossem utilizados para fins científicos e de ensino. Os pacientes que porventura não concordaram em assinar o termo de autorização foram atendidos em psicoterapia da mesma forma por ambas as instituições, mas não fizeram parte da pesquisa. Os dados transpostos ao banco de dados não incluíam o nome dos pacientes.

A análise dos dados foi realizada no programa estatístico SPSS 13. Para caracterização da amostra, todas as variáveis de interesse foram analisadas em termos de levantamento (frequências e porcentagens). Para examinar as relações entre variáveis sociodemográficas e variáveis clínicas e a variável abandono, todas categorizadas, portanto discretas, foi utilizado o teste do qui-quadrado. Foi utilizada a regressão de Poisson para calcular o risco relativo ajustado de abandono, a fim de verificar variáveis relacionadas de forma independente (preditoras) ao abandono. A probabilidade acumulada de abandono de tratamento foi estimada pela curva de Kaplan-Meier. Foram considerados significativos os resultados com valor de $\mathrm{p}$ menores do que 0,05 . 


\section{Resultados}

Foram analisados prontuários de 2.106 crianças. As Tabelas 1 e 2 ilustram a caracterização da amostra. Determinados dados não estavam completos nos prontuários, fazendo com que o número de casos em que não consta a informação seja bastante alto em algumas variáveis.

Das 2.106 crianças que compuseram a amostra total, não há dados sobre o tipo de término de tratamento de 681 pacientes (32,3\% dos casos). A Tabela 3 demonstra a distribuição da amostra quanto ao desfecho do tratamento.

Tabela 1 - Distribuição da amostra quanto às características sociodemográficas

\begin{tabular}{|c|c|c|}
\hline Variável & $\mathbf{n}$ & $\%$ \\
\hline \multicolumn{3}{|l|}{ Sexo } \\
\hline Masculino & 1.391 & 66 \\
\hline Feminino & 715 & 34 \\
\hline Total & 2.106 & 100,0 \\
\hline \multicolumn{3}{|l|}{ Idade } \\
\hline Até 3 anos & 90 & 4,3 \\
\hline 4-6 anos & 510 & 24,3 \\
\hline 7-9 anos & 887 & 42,0 \\
\hline 10-12 anos & 619 & 29,4 \\
\hline Total & 2.106 & 100,0 \\
\hline \multicolumn{3}{|l|}{ Escolaridade } \\
\hline Maternal/creche/berçário & 157 & 7,5 \\
\hline Pré-escola & 246 & 11,8 \\
\hline Alfabetização & 679 & 32,2 \\
\hline $3^{\mathrm{a}}$ série em diante & 683 & 32,4 \\
\hline Não está na escola & 45 & 2,1 \\
\hline Classe especial & 15 & 0,7 \\
\hline Não consta & 281 & 13,3 \\
\hline Total & 2.106 & 100,0 \\
\hline \multicolumn{3}{|l|}{ Configuração familiar } \\
\hline Ambos os pais & 1.214 & 57,7 \\
\hline Apenas mãe & 588 & 27,6 \\
\hline Apenas pai & 33 & 1,6 \\
\hline Mãe e padrasto & 113 & 5,5 \\
\hline Pai e madrasta & 11 & 0,5 \\
\hline Outro familiar & 109 & 5,3 \\
\hline Abrigo de proteção & 6 & 0,3 \\
\hline Não consta & 32 & 1,5 \\
\hline Total & 2.106 & 100,0 \\
\hline \multicolumn{3}{|l|}{ Cidade em que mora } \\
\hline Porto Alegre & 1.772 & 84,1 \\
\hline Região metropolitana & 269 & 12,8 \\
\hline Interior & 52 & 2,5 \\
\hline Não consta & 13 & 0,6 \\
\hline Total & 2.106 & 100,0 \\
\hline
\end{tabular}

Tabela 2 - Distribuição da amostra quanto às características clínicas

\begin{tabular}{lcc}
\hline Variável & $\mathbf{n}$ & $\mathbf{\%}$ \\
\hline Motivo de consulta & & \\
Ansiedade/depressão & 341 & 16,2 \\
Retraimento/depressão & 130 & 6,2 \\
Queixas somáticas & 135 & 6,4 \\
Problemas de relacionamento & 237 & 11,3 \\
Problemas de pensamento & 75 & 3,6 \\
Problemas de atenção & 333 & 15,8 \\
Comportamento desafiador/opositor & 90 & 4,3 \\
Comportamento agressivo & 435 & 20,7 \\
Problemas de aprendizagem & 269 & 12,8 \\
Não consta & 61 & 2,9 \\
Total & 2.106 & 100,0 \\
Fonte de encaminhamento & & \\
Familiares & 197 & 9,3 \\
Escola & 654 & 31,1 \\
Pediatra & 125 & 5,9 \\
Psiquiatra & 100 & 4,7 \\
Neurologista & 150 & 7,1 \\
Outras modalidades médicas & 141 & 6,7 \\
Psicólogo & 215 & 10,2 \\
Pedagogo & 24 & 1,1 \\
Outra instituição & 108 & 5,1 \\
Assistente social & 17 & 0,8 \\
Conselho tutelar & 17 & 0,8 \\
Outros* & 341 & 16,2 \\
Não consta & 187 & 8,9 \\
Total & 2.106 & 100,0 \\
Tempo de atendimento & & \\
Apenas triagem & 102 & 4,8 \\
Menos de 1 mês & 182 & 8,6 \\
1-6 meses & 440 & 20,9 \\
7-12 meses & 156 & 7,4 \\
13-24 meses & 5,5 \\
25-36 meses & 109 \\
Mais do que 37 meses & 100,0 \\
Não consta & & \\
Total & & \\
\hline
\end{tabular}

* Foram agrupados na categoria "outros" todas as fontes de encaminhamento com menos de 15 crianças, dentre elas: fonoaudiólogo, advogado de família, colegas de trabalho dos pais, amigos dos pais, pastores, padres e treinadores de escolinhas de esporte (futebol, balé, natação etc.).

Tabela 3 - Distribuição da amostra quanto ao término de tratamento

\begin{tabular}{lcc}
\hline Tipo de término & n & $\mathbf{\%}$ \\
\hline Não-aderência & 284 & 13,4 \\
Abandono & 793 & 37,7 \\
Alta & 200 & 9,6 \\
Ainda está em atendimento & 148 & 7,0 \\
Não consta & 681 & 32,3 \\
Total & 2.106 & 100,0 \\
\hline
\end{tabular}


Tendo em vista que o objetivo do estudo foi analisar a relação entre as variáveis sociodemográficas e clínicas do paciente e abandono de tratamento, sua amostra final foi composta pelas crianças pertencentes à categoria "abandono" $(\mathrm{n}=793)$ e à categoria "alta" $(\mathrm{n}=200)$. Os dois grupos (alta e abandono) foram comparados, a fim de verificar quais as características relacionadas ao abandono. A amostra final, então, foi composta por 993 crianças. Os resultados quanto à relação entre abandono e variáveis sociodemográficas e clínicas são, a seguir, apresentados.

\section{Abandono e idade}

As variáveis "idade" e "abandono", nesta amostra, não se mostraram associadas (qui-quadrado $=3,476$, graus de liberdade ou $\mathrm{GL}=3, \mathrm{p}=0,324)$.

\section{Abandono e sexo}

Os resultados mostraram associação entre abandono de tratamento e sexo da criança (qui-quadrado $=5,911, \mathrm{GL}=1$, $p=0,015)$. Crianças do sexo masculino apresentam maior risco de abandonar a psicoterapia do que meninas.

\section{Abandono e configuração familiar}

Para calcular a associação entre abandono e configuração familiar, foi necessário alocar em categorias as diversas possibilidades de cuidadores da criança. Como "família" é uma expressão passível de descrições, porém de difícil definição conceitual por assumir diferentes estruturas de agrupamento humano através do tempo ${ }^{23}$, optou-se por realizar os agrupamentos de acordo com três possibilidades. Visto que a literatura mostra relação entre abandono de tratamento e ausência da figura paterna, questionou-se: em qual categoria alocar crianças que moram com padrastos? Da mesma forma, alguns estudos sugerem que as taxas de abandono estão relacionadas a famílias monoparentais; disso adveio a pergunta: a família formada por padrastos e madrastas é conceituada como monoparental?

A primeira possibilidade foi considerar que crianças que moram com a mãe e o padrasto pertencem à categoria "moram apenas com a mãe" e crianças que moram com pai e madrasta pertencem à categoria "moram apenas com o pai". De acordo com essa opção de categorização, não houve associação entre abandono e configuração familiar (qui-quadrado $=3,427$, GL $=3, p=0,330$ ). A segunda possibilidade foi considerar famílias formadas por padrastos e madrastas como famílias nucleares, nas quais estão presentes as figuras materna e paterna. De acordo com essa possibilidade, as variáveis "abandono" e "configuração familiar" não se mostraram associadas (quiquadrado $=1,982, \mathrm{GL}=3, \mathrm{p}=0,576$ ). A terceira possibilidade foi retirar da amostra as crianças que moram com padrastos e madrastas, por se entender que não é possível traçar a natureza singular de cada relação, impossibilitando que se decida com segurança entre as opções “moram apenas com a mãe/pai” e "moram com ambos os pais". Tal medida objetivou evitar o viés de introduzir no cálculo uma variável confundidora. Calculando a relação entre abandono e configuração familiar, definida conforme a terceira possibilidade de categorização (ou seja, excluindo famílias formadas por padrastos e madrastas), também verificou-se que não houve associação (qui-quadrado $=2,576, \mathrm{GL}=3, \mathrm{p}=0,462$ ).

Os resultados indicam, portanto, que, nessa amostra, não houve relação entre abandono de tratamento e configuração familiar.

\section{Abandono e escolaridade da criança}

As variáveis "abandono" e "escolaridade" não se mostraram associadas (qui-quadrado $=6,687, \mathrm{GL}=5, \mathrm{p}=0,245$ ).

\section{Abandono e cidade em que mora}

Os resultados indicam não haver relação entre abandono de tratamento e a cidade em que mora a criança, para a amostra deste estudo (qui-quadrado $=1,727, \mathrm{GL}=2, \mathrm{p}=0,422$ ).

\section{Abandono e motivo de consulta}

A relação entre abandono e motivo de consulta foi calculada de duas formas.

Primeiro, o cálculo foi feito mantendo as nove categorias específicas propostas pelo CBCL. Nessa forma, não houve associação entre abandono e motivo de consulta (qui-quadrado $=4,087, \mathrm{GL}=8, \mathrm{p}=0,849$ ).

Depois, as nove opções de queixas foram recategorizadas de acordo com as categorias amplas propostas pelo CBCL, ou seja: a) competência social = problemas de aprendizagem; b) problemas de comportamento internalizante = ansiedade/depressão, retraimento/depressão e queixas somáticas; c) problemas de comportamento externalizante $=$ comportamento desafiador/opositor e comportamento agressivo; e d) escala neutra $=$ problemas de relacionamento, problemas de pensamento e problemas de atenção. Também não houve associação entre abandono e motivo de consulta (qui-quadrado $=0,922, \mathrm{GL}=3, \mathrm{p}=0,820$ ) no cálculo com as queixas recategorizadas.

Assim, abandono e motivo de consulta não se mostraram associados nessa amostra.

\section{Abandono e fonte de encaminhamento}

Há também duas formas de categorizar a fonte de encaminhamento.

A primeira opção foi realizar os cálculos mantendo as categorias iniciais. Nessa forma, abandono e fonte de encaminhamento mostraram-se associados (qui-quadrado $=23,672$, $\mathrm{GL}=7, \mathrm{p}=0,001)$. Crianças encaminhadas por neurologistas e por psicólogos abandonam menos do que as demais. 
A segunda opção foi realizar os cálculos formando novas categorias para fonte de encaminhamento. Agruparam-se, em uma mesma categoria, denominada "Tratamentos Combinados", todas as crianças encaminhadas para psicoterapia por pediatra, psiquiatra, neurologista, demais modalidades médicas, fonoaudiólogo e pedagogo. Entende-se que esses profissionais encaminham as crianças à psicoterapia a fim de realizarem tratamentos combinados para os problemas do paciente, e objetivou-se verificar se a realização de tratamentos combinados associa-se a abandono de psicoterapia. O resultado apresentou significância limítrofe nessa associação (qui-quadrado $=7,302, \mathrm{GL}=3, \mathrm{p}=0,063$ ), indicando que há possibilidade de as crianças em tratamentos combinados abandonarem menos a psicoterapia, dependendo da amostra.

Assim, há relação entre abandono de tratamento e fonte de encaminhamento para a amostra pesquisada.

\section{Abandono e tempo de atendimento}

Abandono de tratamento mostrou-se associado ao tempo de atendimento para a amostra pesquisada (qui-quadrado $=$ $131,924, \mathrm{GL}=4, \mathrm{p}<0,001)$. Entre o primeiro e o sexto mês de tratamento, há mais risco de a criança abandonar o tratamento. A partir do sétimo mês de tratamento, a criança apresenta menos chances de abandoná-lo do que até o sexto mês. A porcentagem de abandono decai com a passagem do tempo de atendimento, como mostra a Tabela 4.

O abandono de tratamento está, portanto, relacionado ao sexo da criança, à fonte de encaminhamento e ao tempo de atendimento. Para isolar a possível interferência que uma variável pode exercer sobre outra, foi calculado o risco relativo ajustado de abandono. Os resultados indicam que meninos apresentam $10 \%$ mais risco de abandonar o tratamento do que meninas (risco relativo ajustado $=1,10$, IC95\% 1,03-1,19, p = 0,009). Em comparação com encaminhamentos por neurologistas, o risco relativo ajustado de o paciente abandonar o tratamento quando encaminhado por outra fonte mostrou-se significativo:

- No encaminhamento pela família: Risco relativo ajustado $=1,26($ IC95\% $1,05-1,52$, $\mathrm{p}=0,013)$.

- No encaminhamento pela escola: Risco relativo ajustado $=1,22$ (IC95\% 1,03-1,45, $\mathrm{p}=0,019)$.

- No encaminhamento por "outros": Risco relativo ajustado $=1,24$ (IC95\% 1,13-1,59, $\mathrm{p}=0,001)$.

Tais resultados indicam que o paciente encaminhado pela família apresenta $26 \%$ mais risco de abandonar o tratamento do que o paciente encaminhado pelo neurologista. Os encaminhados pela escola têm $22 \%$ mais risco de abandonar do que os encaminhados por neurologista. Os pacientes encaminhados por conselho tutelar, assistente social, pedagogo e pelas fontes explicitadas no rodapé da Tabela 2, por não serem numerosos o suficiente para entrar na análise estatística de forma isolada, foram agrupados na categoria "outros". Nota-se que tal categoria acabou mostrando-se associada ao abandono, já que pacientes derivados para psicoterapia por tais fontes apresentam $24 \%$ mais risco de abandonar do que pacientes encaminhados por neurologista, o que pode ser entendido como resultado espúrio.

Tabela 4 - Variáveis associadas ao abandono de tratamento

\begin{tabular}{lcccc}
\hline Variável & Abandono (\%) & p & Qui-quadrado & GL \\
\hline Sexo & & 0,015 & 5,911 & 1 \\
Masculino & 82,1 & & & \\
Feminino & 75,5 & & & \\
Fonte de encaminhamento & & 0,001 & 23,672 & 7 \\
$\quad$ Familiar & 83,5 & & & \\
Escola & 81,5 & & & \\
Pediatra & 80,0 & & & \\
Psiquiatra & 76,9 & & & \\
Neurologista & 66,7 & & & \\
Outras modalidades médicas & 74,6 & & & \\
Psicólogo & 69,4 & & & \\
Outros & 89,0 & & & \\
Tempo de atendimento & & 0,000 & 131,924 & \\
1-6 meses & 91,8 & & & \\
7-12 meses & 71,0 & & & \\
13-24 meses & 53,5 & & & \\
25-36 meses & 42,4 & & & \\
Mais do que 37 meses & 45,0 & & & \\
\hline GL $=$ graus de liberdade. & & & & \\
\end{tabular}


Em comparação com encaminhamentos por psicólogos, o risco relativo ajustado de o paciente abandonar, quando encaminhado por outra fonte, mostrou-se significativo:

- No encaminhamento pela família:

Risco relativo ajustado $=1,21(\mathrm{IC} 95 \% 1,02-1,42$, $\mathrm{p}=0,026$ ).

- No encaminhamento pela escola:

Risco relativo ajustado $=1,17$ (IC95\% 1,01-1,35, $\mathrm{p}=0,039)$.

- No encaminhamento por "outros":

Risco relativo ajustado $=1,28$ (IC95\% 1,10-1,49, $\mathrm{p}=0,001)$.

Os dados demonstram que o paciente encaminhado pela família apresenta $21 \%$ mais risco de abandonar o tratamento do que o paciente encaminhado pelo psicólogo. Os encaminhados pela escola têm $17 \%$ mais risco de abandonar do que os encaminhados por psicólogos. Pacientes derivados para psicoterapia por "outros" apresentam $28 \%$ mais risco de abandonar do que pacientes encaminhados por psicólogos, outro resultado espúrio.

A curva de Kaplan-Meier demonstra que, uma vez iniciado o tratamento psicoterapêutico (isto é, desconsiderando o mês inicial de avaliação), a probabilidade de o paciente abandonar a psicoterapia no primeiro mês de tratamento é de $13 \%$. Nos primeiros 6 meses de tratamento, a probabilidade de a criança abandonar a psicoterapia é de $54 \%$. Isso significa que nos primeiros 6 meses o paciente tem mais probabilidade de abandonar o tratamento do que seguir em atendimento. Os resultados anteriores mostraram que meninos e crianças que não sejam encaminhadas por neurologistas ou psicólogos aumentam ainda mais essa probabilidade.

\section{Discussão}

Quanto à caracterização da amostra, dados semelhantes foram encontrados em estudos sobre a clientela brasileira de crianças em psicoterapia ${ }^{24-30}$, os quais apontaram maior prevalência de meninos entre a clientela infantil encaminhada para atendimento psicoterapêutico em clínicas de psicologia, em idade escolar, numa faixa etária entre os 6 e os 12 anos, encaminhados pela escola. A literatura ${ }^{31,32}$ aponta que meninos apresentam mais problemas de comportamento externalizante (como agressividade e conduta antissocial) do que meninas, o que causa mais prejuízo no convívio escolar e familiar do que problemas internalizantes (como ansiedade, depressão e retraimento), sendo essa uma possível explicação para que eles sejam encaminhados para psicoterapia com maior frequência do que as meninas. Problemas externalizantes, além de serem mais visíveis, trazem incômodo de forma mais direta aos familiares, justificando a maior demanda de crianças com tais problemas para psicoterapia. A idade de maior procura coincide com a vida escolar e está em consonância com a maior fonte de encaminhamentos para psicoterapia de crianças ser a escola. Possivelmente, a escola esteja mais preparada a detectar problemas infantis do que os pais ou os responsáveis pela criança, razão pela qual alguns sintomas só são percebidos quando a criança entra na escola ${ }^{33}$. Tendo em vista que a escola desempenha importante papel tanto na socialização quanto na aquisição de conhecimentos, espera-se de fato que problemas como comportamento agressivo, problemas de atenção, problemas de aprendizagem e problemas de relacionamento - queixas mais prevalentes nesta amostra - manifestem-se na entrada na escola e sejam mais facilmente percebidos pela escola do que pelos pais, por médicos ou por outros cuidadores. Não obstante, entende-se que o problema escolar pode estar encobrindo outras dificuldades prévias da criança, as quais, com a entrada na escola, passam a ser percebidas em sala de aula, através da dificuldade de formar amizades, aderir a regras ou ajustar-se ao ambiente escolar, por exemplo, justificando assim a maior prevalência de encaminhamentos pela escola ${ }^{28}$.

A distribuição da amostra quanto à configuração familiar reflete a mudança na estrutura familiar observada atualmente. A partir do século XX, verifica-se o surgimento da família "contemporânea": o casamento e a própria família sofrem influências das mudanças sociais; o modelo patriarcal começa a ser questionado; existe maior tendência à redefinição dos papéis masculino e feminino; observa-se a entrada das mulheres no espaço público que anteriormente era ocupado pelo homem. Nesse contexto, o crescimento dos divórcios, a diminuição do número de casamentos formais e a redução do número de filhos aparecem como pontos significativos ${ }^{34}$. O aumento do número de divórcios e de recasamentos e a consequente ruptura do vínculo conjugal do par parental sugerem que as novas estruturas de família podem ser definidas através das variáveis coabitação e consanguinidade ${ }^{35}$. A atual pluralidade de composições familiares foi observada na amostra deste estudo, já que a suposta "família tradicional" - formada por pai, mãe e filhos - representa $57,6 \%$ da amostra da presente pesquisa, enquanto $40,8 \%$ distribuem-se nas demais possibilidades de agrupamentos contemporâneos.

Considerando-se que ambas as instituições localizam-se na cidade de Porto Alegre, torna-se coerente e justificável a maior prevalência de crianças residindo em Porto Alegre ou região metropolitana. $\mathrm{O}$ fato de $2,5 \%$ de crianças atendidas em Porto Alegre serem provenientes do interior do estado levanta a hipótese de que há defasagem entre a demanda por atendimento e a prestação de serviços psicológicos para crianças de baixa e média renda em cidades fora da região metropolitana, obrigando as famílias a percorrerem longos trajetos até a capital para conseguir atendimento para os seus filhos. A porcentagem de crianças encaminhadas para as instituições por psicólogos particulares $(10,2 \%)$ talvez indique que muitas famílias que procuram atendimento em consultórios individuais não podem arcar financeiramente com os honorários cobrados por serviços particulares, sendo derivados a instituições.

A taxa de abandono encontrada neste estudo $(37,7 \%)$ está em consonância com a literatura pesquisada quanto à psicoterapia de crianças ${ }^{3,36}$. Entretanto, desconsiderando os casos em que o tratamento ainda não terminou e aqueles para os quais 
não se pode determinar o tipo de término do tratamento (prontuários em que a informação não consta), a taxa de abandono da amostra pesquisada foi de $62,1 \%$. O estudo de Midgley \& Navridi ${ }^{4}$, realizado nos prontuários do Anna Freud Centre, em Londres, encontrou taxa de abandono de tratamento infantil igual a $60 \%$. Mesmo assim, não é possível determinar se há diferenças ou semelhanças entre a realidade local de Porto Alegre e a realidade internacional quanto às taxas de abandono, em virtude do viés confundidor do uso de distintas definições de "abandono de tratamento" nos estudos.

Quanto às associações entre abandono de tratamento e variáveis sociodemográficas e clínicas, esperava-se (com base na literatura pesquisada) encontrar relação com idade, configuração familiar, escolaridade da criança, distância entre a residência e o local de atendimento e motivo de consulta, variáveis que, nesta amostra, não se mostraram associadas ao abandono. Diversas hipóteses podem ser levantadas com base na ausência de associação estatisticamente significativa. Grande parte dos estudos pesquisados não caracteriza sua amostra e limita-se a demonstrar associações ou não-associações com a variável de interesse (abandono). Portanto, talvez a amostra pesquisada no presente estudo (especificamente crianças atendidas em Porto Alegre, com renda média ou média-baixa, em ambulatórios vinculados a cursos de pósgraduação em psicoterapia psicanalítica) não se assemelhe à amostra pesquisada por outros pesquisadores.

Ademais, para caracterizar a variável "motivo de consulta", o presente estudo coletou queixas alegadas pelos pais, triadores e terapeutas para iniciar a psicoterapia; não fez, entretanto, um entendimento diagnóstico dos problemas da criança. Levanta-se a hipótese de existir aproximação conceitual entre "motivo de consulta" e "diagnóstico" em alguns dos estudos que mostraram associação entre abandono e queixa ${ }^{3,5}$. No estudo realizado com a presente amostra, foi considerada apenas a queixa alegada e não o diagnóstico da criança.

Da mesma forma, diferenças conceituais podem estar envolvidas na ausência de associação estatisticamente significativa entre abandono e configuração familiar para a presente amostra. A definição de "família monoparental" apresenta algumas dificuldades, e as soluções dependem do referencial teórico adotado no estudo. Sugere-se, assim, que os autores explicitem o máximo possível, em seus relatos, as definições adotadas para categorizar suas variáveis, a fim de possibilitar uma comparação mais fidedigna entre os achados e a replicação do estudo em distintas amostras.

O presente estudo encontrou associação entre abandono de tratamento na psicoterapia psicanalítica de crianças e sexo, fonte de encaminhamento e tempo de atendimento.

Meninos apresentam risco ligeiramente superior (10\%) de abandonar seus tratamentos do que meninas, em consonância com a literatura especializada ${ }^{4,5}$. Nos estudos que encontraram associação entre sexo e abandono, o sexo está associado, geralmente, à queixa e ao diagnóstico: em geral, meninos apresentam mais chance de serem levados à terapia por apresentar problemas externalizantes de comportamento e de receberem diagnósticos como funcionamento antissocial e transtornos de conduta. Casos severos como esses apresentam, segundo a literatura, mais risco de abandono de tratamento, colocando, por essa razão, os meninos no chamado "grupo de risco" para abandono de psicoterapia. No presente estudo, não houve, entretanto, associação entre abandono e motivo de consulta, tornando difícil determinar ou propor explicações para o maior risco de abandono em crianças do sexo masculino. $\mathrm{O}$ fato de o estudo não ter encontrado associação entre queixa e abandono não exclui a possibilidade de sexo estar associado à queixa, já que - conforme discutido anteriormente - a presente investigação trabalhou com as queixas levantadas por pais, triadores e terapeutas de forma livre e espontânea e não-estruturada e não trabalhou com diagnósticos. Ademais, o modelo utilizado para categorização das queixas (CBCL) apresenta limitações, como, por exemplo, número restrito de categorias (9), tornando-as demasiadamente abrangentes e pouco específicas. Queixas bastante distintas como fobias e depressões são agrupadas, para fins de análise, na mesma categoria (ansiedade/depressão). Tal inespecificidade pode ter causado um viés de confusão nos achados da pesquisa.

O tratamento combinado mostrou-se, no presente estudo, como fator preventivo de abandono, pois possibilita a compreensão transdisciplinar e mais especializada sobre o problema do paciente, possivelmente aumentando a efetividade do tratamento e satisfazendo melhor as expectativas dos pais. Encaminhamentos por neurologistas atendem a essa perspectiva multidisciplinar. São casos, em geral, com dificuldades de desenvolvimento e que exigem acompanhamento psicológico permanente para a criança e para os pais. Quando os pais percebem e estão de acordo com a necessidade de tratamento, como, em geral, ocorre com crianças com problemas neurológicos, o risco de abandono decai ${ }^{5}$.

$\operatorname{Kazdin}^{5}$ salienta que quanto mais coercitiva for a fonte de encaminhamento, maior a probabilidade de abandono. Fontes coercitivas são aquelas em que o paciente é "mandado" para tratamento (como a escola), em oposição à procura voluntária (como a procura espontânea dos pais). No presente estudo, entretanto, a procura espontânea dos pais aumenta em $26 \%$ o risco de abandono quando comparada à procura por neurologistas, o que contraria Kazdin, mas vai ao encontro das ideias de Midgley \& Navridi ${ }^{4}$. Esses autores discutem que a motivação dos pais para trazer o filho à psicoterapia mostrase ambivalente, e que suas expectativas quanto à terapia são, muitas vezes, irreais, levando ao aumento do abandono.

As crianças encaminhadas por psicólogos podem ser provenientes de avaliação psicológica (na qual ficou constatada a necessidade de psicoterapia) ou de psicólogos que trabalham em consultórios particulares e que encaminham para as instituições as famílias que não apresentam condições de arcar com os honorários particulares. Em qualquer caso, os encaminhamentos por psicólogos permitem uma indicação mais precisa da necessidade de terapia, diminuindo o risco de abandono. A falta de critérios específicos de indicação e contraindicação de tratamento psicoterapêutico mostra-se como um dos fatores responsáveis pelas altas taxas de abandono ${ }^{37}$. 
Outra possível proveniência de crianças encaminhadas por psicólogos é o encaminhamento realizado pelos terapeutas dos pais da criança, por terem percebido a necessidade de atendimento aos filhos de seus pacientes. Nesses casos, os pais trabalham em seus tratamentos sua participação no conflito da criança e contam com auxílio para suportar melhor fases de reação terapêutica negativa da psicoterapia de seus filhos, favorecendo a continuidade do tratamento da criança.

O tempo de tratamento é a variável mais constantemente associada ao abandono de tratamento na literatura. Abandonos de tratamentos são mais comuns nas fases iniciais do atendimento ${ }^{3,11,18}$. A formação de sólida aliança terapêutica entre terapeuta e paciente (e seus familiares) mostra-se como fator protetor para abandono de tratamento ${ }^{7}$, porém essa aliança necessita de tempo de convívio entre os envolvidos para poder se estabelecer. O ponto de corte em que o risco de abandono começa a decair significativamente varia entre os estudos. No presente estudo, o risco de abandono começa a decair significativamente aos 6 meses de tratamento. $\mathrm{O}$ estudo de Urtiaga et al. ${ }^{18}$ mostra que o risco começa a diminuir após a décima sessão (embora o maior risco esteja nas quatro primeiras sessões). Midgley \& Navridi $^{4}$ relataram que a maior parte das crianças cujo término ocorreu por alta recebeu tratamento por mais de 2 anos e revelam que, a cada ano de atendimento que passa, o risco de abandono cai significativamente. Luk et al. ${ }^{3}$ encontraram resultados semelhantes ao da presente investigação: seu estudo demonstrou que, ao final do sexto mês de tratamento, $48 \%$ das crianças tinham abandonado o tratamento, $24 \%$ tinham tido alta e $28 \%$ ainda estavam em atendimento.

Uma vantagem do modelo de fatores de risco, como o empregado neste trabalho, é que a informação pode ser usada para identificar casos de risco para o desfecho, neste caso, o abandono de tratamento. A utilidade dessa informação está na possibilidade de identificar precocemente aqueles casos em risco. Tal monitoramento desde cedo pode se mostrar muito útil na prática clínica. Por exemplo, se um paciente falta a uma ou duas sessões, a reação do terapeuta pode ser muito diferente, dependendo do nível de risco. Para um caso identificado como de risco no início do tratamento, pode-se optar por intervir com mais empenho a fim de prevenir o término prematuro 5 . A identificação precoce de casos de risco permite ao terapeuta empregar qualquer recurso disponível para reter o paciente em tratamento e aumentar suas chances de receber ajuda para suas necessidades.

\section{Conclusões}

Psicoterapeutas psicanalíticos de crianças devem estar atentos, durante os primeiros 6 meses de psicoterapia, para o maior risco que apresentam seus pacientes do sexo masculino e que não vieram consultar encaminhados por neurologistas ou psicólogos de abandonar seus atendimentos. $\mathrm{O}$ abandono do tratamento é frustrante tanto para o terapeuta quanto para o paciente, já que o primeiro sente-se incapaz e desvalorizado profissionalmente ao perder o paciente e o segundo acaba não recebendo a ajuda que procurou na psicoterapia. Entretanto, os psicoterapeutas de crianças precisam estar cientes de que determinados pacientes apresentam mais risco de abandonar o tratamento (meninos encaminhados por outras fontes que não sejam neurologistas e psicólogos) e devem trabalhar mais direta e exaustivamente aspectos da resistência e transferência negativa de tais pacientes e suas famílias durante os primeiros 6 meses de tratamento a fim de evitar que o abandono aconteça. Vencidos os primeiros 6 meses de psicoterapia, o risco de abandonar o tratamento diminui consideravelmente.

Há a hipótese, levantada a partir dos resultados deste trabalho, de que a realização de tratamentos combinados possa contribuir para evitar o abandono de tratamento na psicoterapia psicanalítica de crianças. Tecnicamente, trabalhar em conjunto com profissionais das áreas da neurologia, psiquiatria, fonoaudiologia e pedagogia possivelmente auxilie a diminuir os riscos de abandono de tratamento, pois o tratamento combinado fornece uma visão transdisciplinar do quadro clínico do paciente e possibilita o manejo técnico específico da sintomatologia e sofrimento da criança. Outro manejo técnico possível é identificar precocemente pacientes do grupo de risco de abandono e preparar melhor os pais ou cuidadores desses pacientes para iniciar o tratamento, fazendo maior número de entrevistas iniciais, avaliando melhor os critérios de indicação e contraindicação de psicoterapia psicanalítica e discutindo, desde o princípio da avaliação, aspectos da resistência e transferência negativa dos pais ou familiares da criança, a fim de fortalecer a aliança terapêutica.

Por se tratar de um estudo documental, há limitações inerentes ao método da pesquisa. Os resultados (principalmente quanto ao tipo de término do tratamento e ao motivo de consulta) são derivados de informações constantes nos prontuários dos pacientes, preenchidos subjetivamente por cada terapeuta e dependentes de sua percepção sobre o caso. Ademais, apesar de o estudo ter pesquisado uma grande amostra de crianças em psicoterapia, percebe-se que faltam informações nos prontuários em diversas variáveis de interesse, limitação própria do estudo documental retrospectivo.

Mesmo assim, espera-se que este estudo tenha contribuído tanto para o entendimento teórico quanto técnico da psicoterapia psicanalítica de crianças. Sugere-se que mais estudos como este sejam realizados a fim de aumentar a compreensão clínica que o complexo fenômeno do abandono na psicoterapia psicanalítica de crianças impõe. 


\section{Referências}

1. Botega NJ, Fontanella BJB, Gonçalves EB, Rodrigues RT. Ambulatório de psiquiatria em hospital geral - alguns dados do HC/Unicamp. Rev ABP-APAL. 1988;10(2):57-62.

2. Chen A. Noncompliance in community psychiatry: a review of clinical interventions. Hosp Community Psychiatry. 1991;42(3):282-7.

3. Luk ES, Staiger PK, Mathai J, Wong L, Birleson P, Adler R. Children with persistent conduct problems who dropout of treatment. Eur Child Adolesc Psychiatry. 2001;10(1):28-36.

4. Midgley N, Navridi E. An exploratory study of premature termination in child analysis. J Inf Child Adolesc Psychother. 2006;5:437-59.

5. Kazdin AE. Dropping out of child psychotherapy: Issues for research and implications for practice. Clin Child Psychol Psychiatry. 1996;1(1):133-56.

6. Vargas F, Nunes ML. Razões expressas para o abandono de tratamento psicoterápico. Aletheia 2003;17(18):155-8.

7. Lhullier A, Nunes ML, Horta B. Preditores de abandono de psicoterapia em pacientes de clínica-escola. In: Silvares E, org. Atendimento psicológico em clínicas-escola. Campinas: Alínea; 2006. p. 229-56.

8. Fassino S, Amianto F, Abbate Daga G, Leombruni P. Personality and psychopathology correlates of dropout in an outpatient psychiatric service. Panminerva Med 2007;49(1):7-15.

9. Johansson H, Eklund M. Helping alliance and early dropout from psychiatric outpatient care: the influence of patient factors. Soc Psychiatry Psychiatr Epidemiol. 2006;41(2):140-7.

10. Nickel M, Nickel C, Leiberich P, Mitterlehner F, Forthuber P, Triit K, et al. [Psychosocial characteristics in persons who often change their psychotherapists]. Wien Med Wochenschr. 2004;154(7-8):163-9.

11. Melo APS, Guimarães MDC. Factors associated with psychiatric treatment dropout in a mental health reference center, Belo Horizonte. Rev Bras Psiquiatr. 2005;27(2):113-8

12. Pelkonen M, Marttunen M, Laippala P, Lönnqvist L. Factors associated with early dropout from adolescent psychiatric outpatient treatment. J Am Acad Child Adolesc Psychiatry. 2000;39(3):329-36.

13. Fleitlich-Bilyk B, Goodman R. Prevalence of child and adolescent psychiatric disorders in southeast Brazil. J Am Acad Child Adolesc Psychiatry. 2004;43(6):727-34.

14. Nunes ML, Silva R, Deakin E, Dian S, Campezatto P. Avaliação psicológica e indicação de psicoterapia psicanalítica para crianças. In: Werlang B, Oliveira M, orgs. Temas em psicologia clínica. São Paulo: Casa do Psicólogo; 2006. p. 29-36.

15. Garcia JA, Weisz JR. When youth mental health care stops: therapeutic relationship problems and other reasons for ending youth outpatient treatment. J Consult Clin Psychol. 2002;70(2):439-43.

16. Barbosa JI, Silvares EFM. Uma caracterização preliminar das clínicas-escola de Fortaleza. Estud Psicol (Campinas). 1994;11(3):50-6.

17. Calejon LMC. Estudos com pacientes de clínica psicológica universitária. Mudanças-Psicoter Estud Psicossoc. 1995;3(3/4):235-54.

18. Urtiaga ME, Almeida G, Vianna MED, Santos MV, Botelho S. Fatores preditivos de abandono em psicoterapias: um estudo na clínica Sérgio Abuchaim. J Bras Psiquiatr. 1997;46:279-83.
19. Di Gallo A, Amsler F, Bürgin D. [Discontinuation of treatment in child and adolescent ambulatory psychiatric care in Basel: an evaluation within the scope of quality assurance]. Prax Kinderpsychol Kinderpsychiatr. 2002;51(2):92-102.

20. Shuman AL, Shapiro JP. The effects of preparing parents for child psychotherapy on accuracy of expectations and treatment attendance. Community Ment Health J. 2002;38(1):3-16.

21. Brasil, Estatuto da Criança e do Adolescente. Lei Federal 8.069 de 13 de julho de 1990. Brasília: Diário Oficial da União. 1990. Disponível em: http://www.planalto. gov.br/ccivil 03/LEIS/L8069.htm

22. Gastaud M, Nunes ML. Abandono de tratamento na psicoterapia psicanalítica de crianças [dissertação]. Porto Alegre: PUCRS; 2008.

23. Osório LC. O que é família, afinal? In: Osório LC. Casais e famílias: uma visão contemporânea. Porto Alegre: Artmed; 2002. p.13-23.

24. Campezatto PVM, Nunes MLT. Caracterização da clientela das clínicas-escola de cursos de Psicologia da região metropolitana de Porto Alegre. Psicol Reflex Crit. 2007;20(3):376-88.

25. Marturano EM, Magna JM, Murtha PC. Procura de atendimento psicológico para crianças com dificuldades escolares: um perfil da clientela. Psicol Teor Pesqui. 1993;9(1):207-26.

26. Marturano EM, Toller GP, Elias LCS. Gênero, adversidade e problemas socioemocionais associados à queixa escolar. Estud Psicol (Campinas). 2005;22(4):371-80.

27. Romaro RA, Capitão CG. Caracterização da clientela da clínica-escola de psicologia da Universidade São Francisco. Psicol Teor Prát. 2003;5(1):111-21.

28. Santos MA. Caracterização da clientela de uma clínica da prefeitura de São Paulo. Arqui Bras Psicol. 1990;42(2):79-94.

29. Silvares EFM. É satisfatório o atendimento psicológico nas clinicas-escola brasileiras? Coletâneas ANPEPP. 1996;1(9):137-47.

30. Vanni MG, Maggi $\mathrm{A}$. O que demanda à psicologia na rede pública de saúde em Caxias do Sul? Psico (Porto Alegre). 2005;36(3):299-309.

31. Bolsoni-Silva AT, Marturano EM, Pereira VA, Manfrinato JWS. Habilidades sociais e problemas de comportamento de pré-escolares: comparando avaliações de mães e de professoras. Psicol Reflex Crit. 2006;19(3):460-9.

32. Graminha SSV, Martins MAO. Procura de atendimento psicológico para crianças: características da problemática relatada pelos pais. Psico (Porto Alegre). 1994;25(2):53-79.

33. Gastaud M, Merg M. Diferenças de sexo e idade na psicoterapia de crianças. Rev Soc Psicol RS. In press.

34. Gueiros DA. Família e proteção social: questões atuais e limites da solidariedade familiar. Serviço social e sociedade. São Paulo: Cortez; 2002. p.102-20.

35. Wagner A. Possibilidades e potencialidades da família. A construção de novos arranjos a partir do recasamento. In: Wagner A. Família em cena: tramas, dramas e transformações. Petrópolis: Vozes; 2002. p. 23-38.

36. Kazdin A, Mazurick J. Treatment outcome among children with externalizing disorder who terminate prematurely versus those who complete psychotherapy. J Am Acad Child Adolesc Psychiatry. 1994;33(4):549-57.

37. Hauck S, Kruel L, Sordi A, Sbardellotto G, Cervieri A, Moschetti L, et al. Fatores associados a abandono precoce do tratamento em psicoterapia de orientação analítica. Rev Psiquiatria RS. 2007;29(3):265-7. 